\title{
Ten-Year Outcomes of Transobturator Mid-Urethral Sling for Treatment of Stress Urinary Incontinence in Hong Kong Chinese Women with or without Reconstructive Pelvic Floor Surgery
}

\author{
Toby Yuen Mei Chan, Willy Cecilia Cheon, Yuk Sheung Fan \\ Urogynaecology Team, Department of Obstetrics \& Gynecology, Queen Elizabeth Hospital, Hong Kong, China \\ Email: drymchan@yahoo.com.hk
}

How to cite this paper: Chan, T.Y.M., Cheon, W.C. and Fan, Y.S. (2021) Ten-Year Outcomes of Transobturator Mid-Urethral Sling for Treatment of Stress Urinary Incontinence in Hong Kong Chinese Women with or without Reconstructive Pelvic Floor Surgery. Open Journal of Obstetrics and Gynecology, 11, 1639-1649.

https://doi.org/10.4236/ojog.2021.1111153

Received: October 25, 2021

Accepted: November 27, 2021

Published: November 30, 2021

Copyright $\odot 2021$ by author(s) and Scientific Research Publishing Inc. This work is licensed under the Creative Commons Attribution International License (CC BY 4.0).

http://creativecommons.org/licenses/by/4.0/

\begin{abstract}
Objective: To evaluate the outcomes of transobturator mid-urethral sling (TO-MUS) with or without reconstructive pelvic floor surgery (RPFS) in Chinese women with stress urinary incontinence (SUI) after 10 years. Methods: This was a prospective observational study on Chinese women undergoing the insertion of TO-MUS with or without RPFS. All patients were assessed at 1-year and 10-year by urodynamic study (UDS). Objective cure was defined as the absence of urine leakage during provocative maneuvers on filling cystometry. Data regarding subjective outcome (patient perception), quality of life changes (Urogenital Distress Inventory-short form (UDI-6), Incontinence Impact Questionnaire-short form (IIQ-7)) and adverse events were also collected. Results: Of 104 eligible patients, 99 patients completed the 10-year evaluation. 57 patients (57.6\%) underwent TO-MUS only and 42 patients (42.4\%) underwent TO-MUS with concomitant RPFS. At 10-year follow-up, the overall objective cure rate was $86.9 \%$ and overall subjective cure rate was $80.8 \%$. In TO-MUS only group, the objective and subjective cure rates at 10 -year were $84.2 \%$ and $78.9 \%$ respectively. In TO-MUS with RPFS group, the objective and subjective cure rates at 10 -year were $90.5 \%$ and $83.3 \%$ respectively. Compared TO-MUS only group with TO-MUS with RPFS group, there were no statistically significant differences in objective cure rate $(84.2 \%$ vs. $90.5 \%, \mathrm{p}=0.55)$ and subjective cure rate $(78.9 \%$ vs. $83.3 \%, \mathrm{p}=0.58$ ). Conclusion: TO-MUS is an effective treatment for SUI in Hong Kong Chinese women. Concomitant RPFS during the procedure of TO-MUS does not affect the success.
\end{abstract}




\section{Keywords}

Stress Urinary Incontinence, Urodynamic Stress Incontinence, Mid-Urethral Sling, Transobturator

\section{Introduction}

Stress urinary incontinence (SUI) is defined as the complaint of involuntary leakage of urine upon effort or exertion, or on sneezing or coughing [1]. It is a common and debilitating problem. The life-time risk of a woman having surgery for SUI is estimated to be $13.4 \%$ [2]. The prevalence of stress urinary incontinence in Hong Kong female population is reported to be $40.8 \%$ [3].

With a popular trend of minimally invasive surgery, mid-urethral sling (MUS) has revolutionized the surgical treatment of SUI. First generation of MUS, retropubic mid-urethral sling (RP-MUS), was first introduced in 1996 [4]. Delorme [5] and de Leval [6] developed the second generation: transobturator mid-urethral sling (TO-MUS) which was based on the rationale of avoiding complications such as bladder perforation and visceral damage. TO-MUS was introduced in Hong Kong in 2005.

With an improving life expectancy of Chinese women who are having an average lifespan of 88 years in Hong Kong [7], the prevalence of uterovaginal prolapse and stress urinary incontinence is expected to be increasing over time. Long-term data on the effectiveness, durability and complications of surgical treatment for SUI need to be addressed. The primary outcome of the study was to assess subjective and objective cure rates at 1-year and 10-year after operation of TO-MUS with or without reconstructive pelvic floor surgery (RPFS) in women with SUI by standard pre-designed questions and standard urodynamic investigations. RPFS included anterior or posterior pelvic floor repair (PFR), vaginal hysterectomy $(\mathrm{VH})$ with suspension of vault to uterosacral ligament, laparoscopic sacrocolpopexy. The secondary outcome was to assess the peri-operative and long-term adverse events.

\section{Methods and Materials}

This was a prospective observational study involving women with SUI presenting to out-patient clinic of a local hospital in Hong Kong, Queen Elizabeth Hospital (QEH) from Feb 2005 to Feb 2009. All data was collected prospectively and input to a database established in 1999. Ethical approval was obtained from the hospital ethical committee (Ref: KC/KE-13-0198/ER-3). Informed written consent was obtained from all patients.

The participants were those who complained of predominant SUI symptoms and confirmed to have urodynamic stress incontinence (USI) on urodynamic study (UDS) with failed conservative treatment and undergoing surgery for TO-MUS (inside-out technique, Gynacare, Johnson \& Johnson). 
Preoperatively, all patients were assessed by detailed history with documentation on severity of stress incontinence symptom, frequency/volume chart, urinalysis, urodynamic studies and validated condition specific quality of life (QoL) questionnaires: UDI-6 and IIQ-7. Physical examination was performed with the patient in lithotomy position and pelvic organ prolapse (POP) assessment was described during a maximal Valsalva maneuver according to standard POP quantification system (POP-Q system) [8]. They were assessed by urogynaecologists with UDS using standardized protocol in accordance with good urodynamic practical guideline of International Continence Society (ICS) [9]. USI was defined as involuntary leakage of urine during filling cytometry, upon increased intra-abdominal pressure, in the absence of detrusor contraction. Detrusor overactivity (DO) was defined as involuntary detrusor contractions of more than $15 \mathrm{~cm} \mathrm{H}_{2} \mathrm{O}$ during filling cytometry. For concomitant POP greater than stage 1 according to POPQ system, POP would be reduced by vaginal ring pessary to reduce kinking of urethra before proceeding to cystometry.

Eligible candidates were Chinese, at least 18 years of age, with urodynamically proven USI requesting surgical treatment of SUI after failed conservative management by pelvic floor exercise. Candidates requiring concomitant RPFS were also eligible for the study. Exclusion criteria were as follows: non-Chinese, women with symptoms of overactive bladder syndrome (OAB). OAB was defined as urgency, frequency and nocturia, with or without urge incontinence in the absence of urinary tract infection or obvious pathology. Patients with urodynamically proven DO, mixed urinary incontinence (involuntary loss of urine associated with urgency and also with effort or physical exertion or on sneezing or coughing), a post-void residual urine volume greater than $100 \mathrm{ml}$ and desire for future childbearing were also excluded from the study.

All procedures were performed or supervised by urogynecologists according to the original techniques. Procedures of RPFS included anterior or posterior pelvic floor repair (PFR), vaginal hysterectomy $(\mathrm{VH})$ with suspension of vault to uterosacral ligament, laparoscopic sacrocolpopexy. They were performed in women with symptomatic POP. TO-MUS was inserted after completion of RPFS. Cystoscopy was performed after the procedures to rule out any bladder pathology or urethral damage at the discretion of surgeons' preference. The methods of anesthesia were usually regional or general anesthesia, in accordance with the anesthesiologic requirements and the patients' preference.

After the procedures, indwelling foley catheter would be inserted for drainage of urine and removed on the next day after procedures. Antibiotic prophylaxis (metronidazole $500 \mathrm{mg}$ and Cefuroxime $750 \mathrm{mg}$ intravenously) for three doses were given. Patients were encouraged to void after the removal of foley catheter on the next day (post-operative day 1) when they mobilized well. Patients would be discharged when they voided satisfactorily (residual urine volume less than $150 \mathrm{ml}$ in two consecutive measurements). Post-operative complications such as bladder injury, voiding difficulty, tape exposure, urinary tract infection (with positive culture), wound infection, fever, groin or thigh pain were recorded. 
All patients would be asked to have follow-up at 3 months and 12 months postoperatively, and every $12-18$ months thereafter till 10 years after the procedures. They were assessed subjectively by asking whether their SUI symptoms became "significantly improved or cured" or "no improvement." Symptoms relating to voiding difficulty, urinary urgency and frequency, groin or thigh pain, vaginal pain, or dyspareunia were also assessed. Physical examination was conducted to check for POP or vaginal tape exposure. They were invited for repeating UDS at 1-year and 10-year follow-up for assessing objective outcomes. Changes in condition specific QoL scores was assessed using incontinence-specific questionnaires UDI-6 and IIQ-7 in Chinese version. UDI-6 consisted of six items assessing symptoms of urge, stress and voiding difficulty. The scores of UDI-6 ranged from 0 - 18. IIQ-7 evaluated the impact of urge incontinence on physical activity, travel, social relationships, and emotional health. The IIQ-7 scores ranged from 0 - 21, with higher scores indicating greater symptoms of distress.

Objective cure was defined as the absence of urine leakage during provocative maneuvers on filling cystometry. Subjective cure was defined by patient's perception as there was significant improvement or cured on SUI symptoms. De novo detrusor overactivity (DO) was defined as the new occurrence of DO after insertion of MUS.

Statistical analysis was performed with SPSS v.24. All statistical tests were done using a significance level of $95 \%$. A value for $\mathrm{P}<0.05$ was considered statistically significant. Descriptive statistics of data are presented as mean \pm standard deviation (SD) or number (\%) for continuous variables. Categorical variables were presented as frequency or percentage. Comparisons were performed using Chi square test, Fisher's exact test and McNemar test for categorical variables. Continuous variable was analyzed by independent sample $\mathrm{T}$ test and general linear model (ANOVA). There was no external funding for this study.

\section{Results}

During the study period, 104 eligible patients undergoing TO-MUS were recruited. At the end, 99 patients completed the 10 -year evaluation. 5 patients were reluctant for follow-up assessment.

For patients' demographics (Table 1), the overall mean age of patient at time of surgery was 56.2 years. The mean number of vaginal delivery was 2.9. The mean birth weight of heaviest baby was $3.4 \mathrm{~kg}$. The mean duration of stress incontinence was 6.8 years. 8 patients $(8.1 \%)$ had previous hysterectomy. Of 99 patients, 42 patients $(42.4 \%)$ underwent TO-MUS and concomitant RPFS for SUI and POP, while 57 patients (57.6\%) underwent TO-MUS only. There was no statistically significant difference between TO-MUS only group and TO-MUS with RPFS group in terms of age at time of operation, number of vaginal delivery, birth weight of heaviest baby, duration of SUI or history of previous hysterectomy (Table 1).

The overall objective cure rate was $87.9 \%(87 / 99)$ at 1 -year and $86.9 \%(86 / 99)$ 
Table 1. Patients' demographics.

\begin{tabular}{lcccc}
\hline & ALL & TO-MUS & TO-MUS+ & \\
& $\mathbf{N}=\mathbf{9 9}$ & $\begin{array}{c}\text { only } \\
\mathbf{N}=\mathbf{5 7}\end{array}$ & $\begin{array}{c}\text { RPFS } \\
\mathbf{N}=\mathbf{4 2}\end{array}$ & P value \\
\hline Age at time of operation (years) & $56.2 \pm 9.9$ & $54.7 \pm 8.8$ & $58.2 \pm 11.1$ & 0.08 \\
Previous vaginal delivery & $2.9 \pm 1.4$ & $2.7 \pm 1.3$ & $3.1 \pm 1.6$ & 0.18 \\
Birth weight of heaviest baby (kg) & $3.4 \pm 0.6$ & $3.4 \pm 0.5$ & $3.4 \pm 0.6$ & 0.78 \\
Duration of SUI (years) & $6.8 \pm 5.6$ & $7.4 \pm 5.9$ & $5.9 \pm 6.0$ & 0.18 \\
Previous hysterectomy & 8 & $6(10.5 \%)$ & $2(4.7 \%)$ & 0.46 \\
\hline
\end{tabular}

SUI: stress urinary incontinence; TO-MUS: transobturator midurethral sling; RPFS: reconstructive pelvic floor surgery. Data are shown as No. (\%), mean \pm standard deviation; $\mathrm{p}<0.05$ was considered statistically significant.

at 10-year follow-up. There was no statistically significant difference in overall objective cure rate at 1 -year and 10 -year $(87.9 \%$ vs. $86.9 \%, \mathrm{p}=1.00)$. The objective cure rate at 1 -year was $86.0 \%$ (49/57) for TO-MUS only group and $90.5 \%$ (38/42) for TO-MUS with RPFS group. At 10-year, the objective rate was $84.2 \%$ (48/57) for TO-MUS only group and 90.5\% (38/42) for TO-MUS with RPFS group. There was no statistically significant difference in objective cure rate between both groups ( $86 \%$ vs. $90.5 \%, \mathrm{p}=0.55)$ at 1 -year and $(84.2 \%$ vs. $90.5 \%, \mathrm{p}=$ 0.55 ) at 10 -year (Table 2).

The overall subjective cure rate was $91.9 \%$ (91/99) at 1-year and $80.8 \%(80 / 99)$ at 10 -year. There was significant difference in overall subjective cure rate at 1 -year and 10 -year $(91.9 \%$ vs. $80.8 \%, \mathrm{p}=0.013)$. The subjective cure rate at 1 -year was $93 \%$ (53/57) for TO-MUS only group and 90.5\% (38/42) for TO-MUS with RPFS group. At 10-year, the subjective cure rate was $78.9 \%$ (45/57) for TO-MUS only group and $83.3 \%$ (35/42) for TO-MUS with RPFS group. There was no statistically significant difference in subjective cure rate between the two groups $(93 \%$ vs. $90.5 \%, \mathrm{p}=0.72)$ at 1 -year and $(78.9 \%$ vs. $83.3 \%, \mathrm{p}=0.58)$ at 10-year (Table 2).

In the study groups, the overall rate of de novo DO was found in $20.2 \%$ (20/99) of patients at 1-year and $16.2 \%$ (16/99) of patients at 10-year. There was no statistically significant difference between 1-year and 10-year results $(20.2 \%$ vs. $16.2 \%, p=0.45$ ) (Table 2). Among patients with de novo DO, $35 \%$ of patients presented with wet-OAB and were given with anti-muscarinic treatment with satisfactory control of the symptoms, $65 \%$ of patients preferred conservative management since symptoms were mild and not troublesome.

For TO-MUS only group, the mean QoL scores of both UDI-6 and IIQ-7 improved significantly over 10 years $(\mathrm{p}=0.000)$. Compared with pre-operative status of TO-MUS with RPFS, the mean IIQ-7 scores showed significant improvement $(\mathrm{p}=0.000)$ but not the mean UDI-6 scores $(\mathrm{p}=0.075)$ over ten-year period (Table 3 ).

Regarding post-operative complications, 1 patient (1\%) from TO-MUS only 
Table 2. Outcomes at 1-year and 10-year follow-up.

\begin{tabular}{|c|c|c|c|c|c|c|c|c|}
\hline Outcome & $\begin{array}{c}1 \text {-year } \\
\text { follow-up }\end{array}$ & & & & $\begin{array}{c}10 \text {-year } \\
\text { follow-up }\end{array}$ & & & \\
\hline & $\begin{array}{c}\text { Total } \\
\mathrm{N}=99\end{array}$ & $\begin{array}{c}\text { TO-MUS } \\
\text { only } \\
\mathrm{N}=57\end{array}$ & $\begin{array}{c}\text { TO-MUS+ } \\
\text { RPFS } \\
\mathrm{N}=42\end{array}$ & $\mathrm{P}$-value & $\begin{array}{c}\text { Total } \\
\mathrm{N}=99\end{array}$ & $\begin{array}{c}\text { TO-MUS } \\
\text { Only } \\
\mathrm{N}=\mathbf{5 7}\end{array}$ & $\begin{array}{c}\text { TO-MUS+ } \\
\text { RPFS } \\
\mathrm{N}=42\end{array}$ & $\mathrm{P}$-value \\
\hline Objective & 87 (87.9\%) & $49(86 \%)$ & $38(90.5 \%)$ & 0.55 & $86(86.9 \%)$ & $48(84.2 \%)$ & $38(90.5 \%)$ & 0.55 \\
\hline Subjective & 91 (91.9\%) & $53(93.0 \%)$ & $38(90.5 \%)$ & 0.72 & $80(80.8 \%)$ & $45(78.9 \%)$ & $35(83.3 \%)$ & 0.58 \\
\hline Denovo DO & $20(20.2 \%)$ & $8(14 \%)$ & $12(28.6 \%)$ & 0.08 & $16(16.2 \%)$ & $8(14 \%)$ & $8(19.1 \%)$ & 0.50 \\
\hline
\end{tabular}

TO-MUS: transobturator midurethral sling; RPFS: reconstructive pelvic floor surgery; DO: detrusor overactivity. Data are shown as No. (\%); $\mathrm{p}<0.05$ was considered statistically significant.

Table 3. Results of UDI-6 and IIQ-7 scores over time.

\begin{tabular}{cccccc}
\hline & & Pre-operative & 1 -year & 10 -year & p-value \\
\hline TO-MUS only & UDI-6 & $7.1 \pm 3.0$ & $0.8 \pm 1.2$ & $2.2 \pm 1.9$ & 0.000 \\
& IIQ-7 & $5.7 \pm 4.8$ & $0.1 \pm 0.3$ & $0.7 \pm 1.5$ & 0.000 \\
\hline TO-MUS + RPFS & UDI-6 & $6.0 \pm 3.3$ & $1.1 \pm 2.1$ & $1.9 \pm 1.7$ & 0.075 \\
& IIQ-7 & $5.4 \pm 5.4$ & $0.4 \pm 1.5$ & $0.6 \pm 1.7$ & 0.000 \\
\hline
\end{tabular}

UDI6: Urogenital Distress Inventory 6; IIQ7: Incontinence Impact Questionnaire 7; TO-MUS: transobturator midurethral sling; RPFS: reconstructive pelvic floor surgery. Data are shown as mean \pm standard deviation; $\mathrm{p}<0.05$ was considered statistically significant.

group had developed voiding difficulty with post-void residual urine more than $150 \mathrm{ml}$ immediately after operation (Table 4). The patient also developed lower limb weakness and impaired sensation below T10 after spinal anesthesia. Anesthetists and orthopedic surgeons were consulted. Urgent magnetic resonance imaging showed no evidence of haematoma at spinal cord. Bladder training was started on post-operative Day 8 when sensation of lower limb resumed. Finally, the patient was discharged on post-operative Day 14 with minimal residual urine. Urodynamic test at 10-year showed peak flow rate $17 \mathrm{ml} / \mathrm{s}$ with voided volume of $495 \mathrm{ml}$ and residual urine of $0 \mathrm{ml}$.

2 patients $(2 \%)$ complained of groin pain within 3 months after operation (Table 4). Both had TO-MUS insertion with concomitant RPFS. Groin pain improved six months post-operatively with analgesics and physiotherapy. No long-term groin or thigh pain was reported for both patients.

There were 2 patients (2\%) with tape exposure (one from each group) (Table 4). The patient in the TO-MUS only group reported dyspareunia 3 months after the procedure. Clinical examination showed $0.5 \mathrm{~cm}$ tape exposure. The extruded tape was removed by excision under local anesthesia and wound was closed with absorbable stitches. No tape exposure was detected at subsequent follow-up two months after tape excision. There was no complaint of stress urinary incontinence. No recurrent stress incontinence was detected on UDS at 10-year follow-up. 
Table 4. Post-operative complications.

\begin{tabular}{|c|c|c|c|c|}
\hline Complications & $\begin{array}{c}\text { Total } \\
\mathrm{N}=99\end{array}$ & $\begin{array}{l}\text { TO-MUS only } \\
\quad \mathrm{N}=57\end{array}$ & $\begin{array}{c}\text { TO-MUS + RPFS } \\
\mathrm{N}=42\end{array}$ & $P$ value \\
\hline Fever (within first 48 hours) & $19(19.2 \%)$ & $4(7.0 \%)$ & $15(35.7 \%)$ & 0.000 \\
\hline Urinary tract infection & $3(3 \%)$ & 0 & $3(7.1 \%)$ & 0.07 \\
\hline Voiding difficulty & 1 & $1(1.0 \%)$ & 0 & 0.42 \\
\hline $\begin{array}{l}\text { Post-operative thigh pain or } \\
\text { groin pain ( } 3 \text { months) }\end{array}$ & $2(2.0 \%)$ & 0 & $2(2.0 \%)$ & 0.18 \\
\hline Tape exposure & $2(2.0 \%)$ & $1(1.0 \%)$ & $1(1.0 \%)$ & 1.00 \\
\hline
\end{tabular}

TO-MUS: transobturator midurethral sling; RPFS: reconstructive pelvic floor surgery. Data are shown as No. (\%); $\mathrm{p}<0.05$ was considered statistically significant.

For the other patient in TO-MUS with RPFS group, tape exposure was found at 5 -year follow-up after operation. The patient preferred conservative management as she was asymptomatic. On subsequent follow-up at 10-year, spontaneous healing with no tape exposure was found.

Post-operative fever (within first 48 hours) was found in 19 patients $(19.2 \%, 4$ in TO-MUS only group and 15 in TO-MUS with RPFS group), who were treated successfully with antibiotics and antipyretics. There was statistically significant difference in post-operative fever between TO-MUS only group and TO-MUS with RPFS group (7\% vs. $35.7 \%, \mathrm{p}=0.000$ ) (Table 4).

There was no statistically significant difference between both groups in terms of urinary tract infection ( $0 \%$ vs. $7.1 \%, p=0.07)$, voiding difficulty $(1.0 \%$ vs. $0 \%$, $\mathrm{p}=0.42)$, post-operative groin pain ( $0 \%$ vs. $2 \%, \mathrm{p}=0.18)$ and tape exposure $(1 \%$ vs. $1 \%, \mathrm{p}=1.00)$ (Table 4 ). No bladder damage, chest or wound infection, or deep vein thrombosis was reported.

\section{Discussion}

Our study reported subjective, objective and urodynamic outcomes of TO-MUS in women with or without RPFS across the 10-year follow-up. The challenge of this study was to collect the representative number of patients with long-term data. The default rate was $5.1 \%$ which was low and acceptable over 10 years when compared with default rate $5 \%-51 \%$ in other studies [10] [11] [12] [13] [14].

The overall objective and subjective cure rates at 10 -year were $86.9 \%$ and $80.8 \%$ in our study, which were similar with the results in the study by Starvo et al. [11]. The author retrospectively assessed 124 women 7 years after TO-MUS showed an overall objective cure rate of $81.5 \%$ by cough stress test and subjective cure rate of $83.5 \%$ [11].

In our study, the objective cure rates were $86 \%$ at 1 -year and $84.2 \%$ at 10 -year in TO-MUS only group; $90.5 \%$ at 1 -year and $90.5 \%$ at 10 -year in TO-MUS with RPFS group. In 2015, Law et al. [15] evaluated 218 women having insertion of MUS with or without concomitant RPFS and showed comparable results. The 
5-year objective cure rate was $80.3 \%$ in MUS only group and $85.7 \%$ in MUS with concomitant RPFS. Performing reconstructive pelvic floor surgery during MUS operation was found to be a reliable procedure while having no detrimental impact on the success of MUS.

The subjective cure rates were $93 \%$ at 1 -year and $78.9 \%$ at 10 -year in TO-MUS only group; while $90.5 \%$ at 1 -year and $83.3 \%$ at 10 -year in TO-MUS with RPFS group but they did not reach any statistically significant difference. The significant difference in the overall subjective cure rate as shown by patient's perception of cure or improvement at 1-year and 10-year (91\% vs. $80.8 \%, p=0.013$ ) may be related to the fact that there was lack of standardized validated subjective symptom assessment tool and small sample size. In future study, subjective outcomes can be assessed by validated tools like Patient Global Impression of improvement (PG-I) scale (a 7-point scale with a range of response form 1 "very much improved" to 7 "very much worse") and patient satisfaction scale (a single, self-answered, Likert-type scale of 0 - 10 that grades the patients' satisfaction).

Regarding measurement of incontinence-related QoL, there was significant reduction in mean IIQ-7 scores in women having TO-MUS with RPFS ( $\mathrm{p}=$ 0.000 ) or without RPFS ( $\mathrm{p}=0.000$ ) during 10-year study period. It suggested that TO-MUS improved SUI symptoms and had positive impact on daily activities and emotional health in long term [16] [17]. On the other hand, TO-MUS with RPFS group had an insignificant change in mean UDI-6 scores at 10 years $(\mathrm{p}=0.075)$ when compared with TO-MUS only group $(\mathrm{p}=0.000)$. It is understood that UDI- 6 assesses 3 main domains related to urge, stress and voiding difficulty. It may be related to the recurrence of POP and high percentage of de novo DO in TO-MUS with RPFS group. The benefit of RPFS may not be assessed appropriately by UDI-6 questionnaire. In future, assessment on pelvic organ prolapse will require condition-specific validated tools: Pelvic Floor Distress Inventory (PFDI-20) or Pelvic Floor Impact Questionnaire (PFIQ-7).

The most commonly encountered morbidity was de novo DO after TO-MUS (20.2\% at 1-year and $16.2 \%$ at 10 -year) which was similar to that reported in the literature [10] [12] [13]. This may be explained by the fact that UDS is used to define DO instead of OAB symptoms in our study. DO is an urodynamic diagnosis, which may not correlate well with symptoms of OAB and leads to over-estimation of the true picture. This is important for pre-operative counselling because post-operative de novo DO affects patient satisfaction and healthy-related quality of life.

In the literature, the incidence of complication of TO-MUS was reported with a wide range $(0.8 \%-33 \%)$ [18]. In the present study, the overall complication rate was $27.2 \%$ (27/99) which included voiding difficulty (1\%), groin pain $(2 \%)$, tape exposure $(2 \%)$, urinary tract infection $(3 \%)$ and post-operative transient fever (19.2\%).

Groin pain mostly occurs in early post-operative period and will be significantly reduced over time [19]. Persistence groin pain is not common with reported rate up to $0.6 \%$ [10] [12] [13]. There was $2 \%$ rate of tape exposure in our 
study which was comparable with $0.8 \%-5 \%$ in other studies [10] [12] [14]. The condition can be treated with partial excision of exposed tape without compromising the continence outcome.

Bedsides transient post-operative fever and urinary tract infection, there was no major peri-operative and post-operative morbidity associated with TO-MUS in our study. Higher chance of transient post-operative fever in TO-MUS with RPFS group could be related to longer operation time and possibility of tissue inflammation resulted from more tissue dissection.

The strength of the study is the prospective design with low "drop-out" rate (5.1\%) over 10-year and the use of urodynamic study for assessment of objective success. Validated questionnaires (UDI-6, IIQ-7) in Chinese version are used to assess the effect of the operation on quality of life of patients.

We acknowledge the limitations of our study. There is a lack of validated assessment tools for assessment of patients' subjective cure and lack of blinding when assessing the objective outcome. Also, there is a possibility of sampling bias due to the heterogeneous data arising from different degree of pelvic organ prolapse and different types of prolapse surgery. Future prospective study including large study population and use of validated assessment tools on patients' satisfaction, subjective outcome and the effect of pelvic organ prolapse are required to verify our results.

\section{Conclusion}

Our ten-year study demonstrates that transobturator mid-urethral sling is an effective and safe treatment option for stress urinary incontinence in Chinese women. Performing reconstructive pelvic floor surgery at the same setting of insertion of transobturator mid-urethral sling does not compromise the outcomes.

\section{Conflicts of Interest}

The authors declare no conflict of interest.

\section{References}

[1] Haylen, B.T., de Ridder, D., Freeman, R.M., Swift, S.E., Berghmans, B., Lee, J., et al. (2010) An International Urogynecological Association (IUGA) / International Continence Society (ICS) Joint Report on Terminology of Female Pelvic Floor Dysfunction. Neurourology and Urodynamics, 29, 4-20. https://doi.org/10.1002/nau.20798

[2] Wu, J.M., Matthews, C.A., Conover, M.M., Pate, V. and Jonsson Funk, M. (2014) Life-Time Risk of Stress Urinary Incontinence or Pelvic Organ Prolapse Surgery. Obstetrics \& Gynecology, 123, 1201-1206. https://doi.org/10.1097/AOG.0000000000000286

[3] Wong, T., Lau, B.Y., Mak, H.L., et al. (2006) Changing Prevalence and Knowledge of Urinary Incontinence among Hong Kong Chinese Women. International Urogynecology Journal and Pelvic Floor Dysfunction, 17, 593-597. https://doi.org/10.1007/s00192-006-0072-3

[4] Ulmsten, U., Henriksson, L., Johnson, P., et al. (1996) An Ambulatory Surgical Procedure under Local Anesthesia for Treatment of Female Urinary Incontinence. 
International Urogynecology Journal, 7, 81. https://doi.org/10.1007/BF01902378

[5] Delorme, E. (2001) Transobturator Urethral Suspension: Mini-Invasive Procedure in the Treatment of Stress Urinary Incontinence in Women. Progrès en Urologie, 11,1306 .

[6] De Leval, J. (2003) Noval Surgical Technique for the Treatment of Female Stress Urinary Incontinence: Transobturator Vaginal Tape Inside-Out. European Urology, 44, 724. https://doi.org/10.1016/j.eururo.2003.09.003

[7] Hong Kong Census 2018. https://chp.gov.hk/

[8] Bump, R.C., Mattiasson, A., Bo, K., et al. (1996) The Standardization of Terminology of Female Pelvic Organ Prolapse and Pelvic Floor Dysfunction. American Journal of Obstetrics \& Gynecology, 175, 10-17. https://doi.org/10.1016/S0002-9378(96)70243-0

[9] Schafer, W., Abram, P., Liao, L., et al. (2002) Good Urodynamic Practices: Uroflowmetry, Filling Cystometry, and Pressure-Flow Studies. Neurourology and Urodynamics, 21, 261-274. https://doi.org/10.1002/nau.10066

[10] Ulrich, D., Tammaa, A., Holbfer, S., Trutnovsky, G., Radisic, V.B., Tamussino, K., et al. (2016) Ten-Year Follow-Up after Tension Free Vaginal Tape-Obturator Procedure for Stress Urinary Incontinence. The Journal of Urology, 196, 1201-1206. https://doi.org/10.1016/j.juro.2016.05.036

[11] Athanasiou, S., Grigoriadis, T., Zacharakis, D., Skampardonis, N., Lourantou, D. and Antsaklis, A. (2014) Seven Years of Objective and Subjective Outcomes of Transobturator (TVT-O) Vaginal Tape: Why Do Tapes Fail? International Urogynecology Journal, 25, 219-225. https://doi.org/10.1007/s00192-013-2186-8

[12] Montera, R., Miranda, A., Plotti, F., Terranova, C., Luvero, D., Capriglione, S., et al. (2018) Anterior Colporrhaphy plus Inside-Out Tension-Free Vaginal Tape for Associated Stress Urinary Incontinence and Cystocele: 10-Year Follow Up Results. Neurourology and Urodynamics, 37, 1144-1151. https://doi.org/10.1002/nau.23439

[13] Sertia, M., Braga, A., Athanasiou, S., Tommaselli, G., Caccia, G., Torella, M., et al. (2017) Tension-Free Vaginal Tape-Obturator for Treatment of Pure Urodynamic Stress Urinary Incontinence: Efficacy and Adverse Effect at 10-Year Follow-Up. European Urology, 71, 674-679. https://doi.org/10.1016/j.eururo.2016.08.054

[14] Serati, M., Braga, A., Caccia, G., Torella, M., Ghezzi, F., Salvatore, S., et al. (2020) TVT-O for Treatment of Pure Urodynamic Stress Urinary Incontinence: Efficacy and Adverse Effects at 13-Years Follow-Up. Neurourology and Urodynamics, 39, 1423-1429. https://doi.org/10.1002/nau.24358

[15] Law, S.M., Cheung, Y.K., Chung, K.H. and Chan, S.C. (2015) Efficacy and Outcomes of Transobturator Tension-Free Vaginal Tape with or without Concomitant Pelvic Floor Repair Surgery for Urinary Stress Incontinence: Five-Year Follow-Up. Hong Kong Medical Journal, 21, 333-338.

[16] Huang, W.C., Lau, H.H. and Su, T.H. (2018) Did Surgical Failure and Complications Affect Incontinence-Related Quality of Life on Women after Trans-Obturator Sling Procedure? Taiwanese Journal of Obstetrics \& Gynecology, 57, 295-299. https://doi.org/10.1016/j.tjog.2018.02.020

[17] Coskun, B., Aksakal, O.S., Coskun, B., Yurtcu, E., Akkurt, M.O., Kafadar, O., et al. (2018) The Impact of Concurrent Pelvic Organ Prolapse Reconstructive Surgery on Midurethral Sling Procedure Outcome. Via Medica Journals, 89, 190-195. https://doi.org/10.5603/GP.a2018.0032

[18] Lin, Y.H., Lee, C.K., Chang, S.D., Chien, P.C., Hsu, Y.Y. and Tseng, L.H. (2021) 
Focusing on Long-Term Complications of Mid-Urethral Slings among Women with Stress Urinary Incontinence as a Patient Safety Improvement Measure. Medicine, 100, 23-34. https://doi.org/10.1097/MD.0000000000026257

[19] Reham, F., Yahia, M., Ahmed, T. and Sherine, H. (2017) Uroflowmetric Changes, Success Rate and Complications Following Tension-Free Vaginal Tape Obturator (TVT-O) Operation in Obese Females. European Journal of Obstetrics \& Gynecology and Reproductive Biology, 214, 6-10.

https://doi.org/10.1016/j.ejogrb.2017.04.038 